\title{
DE
}

BULGARIAN ACADEMY OF SCIENCES

CYBERNETICS AND INFORMATION TECHNOLOGIES • Volume 14, No 3

Sofia $\bullet 2014$

Print ISSN: 1311-9702; Online ISSN: 1314-4081

DOI: $10.2478 /$ cait-2014-0033

\section{A Large-Scale MIMO Precoding Algorithm Based on Iterative Interference Alignment}

\author{
Jing Jiang, Wei $L v$ \\ Xi'an University of Posts \& Telecommunications, Xi'an, CO 710061 China \\ Emails: jiangjing18@gmail.com longweiba3000@sina.com
}

\begin{abstract}
The performance of Large-scale MIMO system is degraded by Pilot Contamination. In order to reduce Pilot Contamination, a downlink precoding algorithm is put forward, based on Interference Alignment (IA). The main idea of this algorithm is aligning the pilot contamination and inter-cell interference to the same null space in order to acquire the maximal degrees of freedom. Then the downlink receiving precoding matrix is solved with respect to a maximal SINR (Signal Interference Noise Ratio) criterion. Exploiting the channel reciprocity and an iterative process, Base station and User Equipment switch transmitting and receiving roles in the uplink and downlink, the precoding matrices of the Base station and User Equipment is gradually updated until convergence. Finally, the simulation results have shown that the algorithm proposed can efficiently mitigate the impact of pilot contamination and outperform some popular precoding algorithms, e.g., MF precoding algorithm and MMSE precoding algorithm. When the number of antennas increases, the performance of the proposed algorithm will be greatly improved.
\end{abstract}

Keywords: Massive MIMO, large-scale MIMO, precoding, pilot contamination, interference alignment.

\section{Introduction}

Large-scale MIMO or Massive MIMO is an emerging research topic of the fifth generation broadband wireless systems, which scales up MIMO to possible orders of magnitude compared to current systems [1]. Antenna arrays with hundreds of antenna elements provide enormous large-scale array gains and interference suppression gains [2-3]. But the performance gain of the Large-scale MIMO relies on good enough channel knowledge of the downlink and uplink channel. 
In the current system, the transmitter transmits pilots, and then the receiver estimates the channel impulse responses from the received pilots and feedbacks the channel information to the transmitter. In the Large-scale MIMO system, the amount of channel information is greatly increased with antennas increasing, the channel state information needs vast physical resources to feedback. To avoid mass feedback overhead, Large-scale MIMO system calculates the downlink precoding matrix, utilizing the channel reciprocity in a TDD working mode. In it the user equipment transmits the uplink pilots, the base station estimates the uplink channel information and calculates the precoding matrix, based on the estimated uplink channel information instead of the downlink channel information feedback. This scheme reduces the signaling overhead and simplifies the feedback process, but the channel estimation of the local cell may be interfered by non-orthogonal uplink pilots of adjacent cells in an undesirable manner and result in inaccurate calculating of the downlink precoding matrix. The performance of the Large-scale MIMO is evenly degraded due to the impact of Pilot Contamination caused by the uplink interference from non-orthogonal pilots of other cells [4-5].

Thus the key issue for a Large-scale MIMO system is how to reduce the pilot contamination [6-10]. The authors in [6] considered that the uplink channel estimation was contaminated by non-orthogonal pilots from different cells and analyzed the performance degradation caused by the pilot contamination. A multicell MMSE precoding was proposed to mitigate the pilot contamination which minimized the interference from uplink pilots of other cells. Reference [7] studied the effects of shifting the time location of pilots in neighboring cells and proved that it could obtain a better channel estimation because of the inter-cell interference reduction. [8] considered a downlink Pilot Contamination Precoding (PCP) designing in the finite antenna regime. It formulated the downlink PCP design as a source optimization problem to maximize the minimum SINR among all users subject to network sum power constraints. The simulation results showed that in a practical scenario, the proposed optimal and suboptimal PCP give very large gains over conventional LSASs with 100 transmitting antennas. In [9] Pilot Contamination Precoding (PCP) is designed, in which each base station linearly combines slow-fading coefficients between the terminals and the base stations to a slow-fading coefficients matrix. The precoding matrix is the product of the slowfading coefficients matrix and a well known single-cell precoding matrix. It can achieve the capacity lower bound of PCP which can efficiently eliminate the intercell interference in Large-scale MIMO systems. In [10] appropriate power allocation algorithms are proposed under the circumstance of avoiding simultaneous non-orthogonal transmissions from adjacent cells. The techniques proposed are especially beneficial to users in unfavorable locations that would otherwise suffer of low SINR.

Interference Alignment (IA) is a promising technique to efficiently mitigate interference and achieve the maximum spatial degrees of freedom in wireless communication systems [11-14]. In [11] the authors have proved that linear schemes of interference alignment sufficed to achieve all the degrees of freedom and deduced the basic principles of interference alignments in conditions of multi- 
cell and single-user in every cell. In [12] examples of iterative algorithms that utilized the reciprocity of wireless channel to achieve interference alignment with only local channel knowledge at each node are provided. The algorithm can acquire the minimal leakage of transmitting signals and the maximum degree of freedom of desired receiving signals. The numerical results proved that this algorithm could achieve theoretically optimal performance. To efficiently mitigate multi-cell interferences, a simple coordinated Zero-Forcing (ZF) scheme was proposed in [13]. It reduced both ICI (Inter Carrier Interference) and IUI (Inter Users Interference) simultaneously and achieved the optimal Degrees of Freedom (DoF) in the multiple-input single-output interfering broadcast channels. Since this scheme considered a user with a single antenna, it only designed the transmitting beam forming vectors. In [14] the authors have developed an Interference Alignment (IA) technique for a downlink cellular system. The proposed scheme could be implemented with minimal changes to an existing cellular system where the feedback mechanism (within a cell) is already being considered for supporting multi-user MIMO. Moreover, it could provide a substantial gain especially when the interference from a dominant interferer is significantly stronger than the remaining interference.

In this paper the interference alignment will be applied to Large-scale MIMO for the first time. Based on the basic theory of interference alignment [12], the goal is designing the receiving precoding matrix to minimize pilot contamination in addition to the interferences from other cells, meanwhile maximizing the degrees of space freedom for the desired receiving signals. Then exploiting the channel reciprocity, the transmitter and receiver iteratively switch roles to solve the receiving precoding matrix until the precoding matrices are updated to convergence. It is theoretically proved that the maximal SINR in the downlink is equal to achieving a maximal SLNR in the uplink. Another idea of this paper is to replace the estimated channel and the pilot contamination channel as the true channel in the solving process, so that it can more efficiently mitigate the impact of pilot contamination on Large-scale MIMO precoding. Numerical results have proved that the proposed algorithm could outperform some of the popular techniques, e.g., MF algorithm and multi-cell MMSE precoding algorithm.

\section{System model}

In Large-scale MIMO system, I cells compose the cellular network, each cell consists of a base station with $M$ antennas and $K$ users with $N$ receiving antennas. To analyze the pilot contamination, it is assumed that there are $L$ cells reused by the same pilots. In addition, OFDM symbol $t$ and subcarriers $\omega$ of the uplink training resource will all be assigned to user $k$ of per cell.

As previously mentioned, Large-scale MIMO system works in TDD mode. The reciprocity for uplink and downlink exists, i.e., $\boldsymbol{H}=\overline{\boldsymbol{H}}^{H}, \boldsymbol{H}^{H}$ is the conjugate transpose of matrix $\boldsymbol{H}$. The downlink channel $\boldsymbol{H}$ is equal to the transposition of uplink channel training $\hat{\boldsymbol{H}}$ in the coherence time. 
As shown in Fig. 1, the receiving signal $\overline{\boldsymbol{y}}_{j}$ of the base station of cell $j$ for OFDM symbol $t$ and subcarriers $\omega$ is

$$
\overline{\boldsymbol{y}}_{j}=\sqrt{p_{r} \tau} \overline{\boldsymbol{H}}_{j j} \overline{\boldsymbol{x}}_{j}+\sum_{l=1, l \neq j}^{L} \sqrt{p_{r} \tau} \overline{\boldsymbol{H}}_{j l} \overline{\boldsymbol{x}}_{j}+\sum_{i=1, i \neq l}^{I} \sqrt{p_{r} \tau} \overline{\boldsymbol{H}}_{j i} \overline{\boldsymbol{x}}_{i}+\overline{\boldsymbol{n}}_{j},
$$

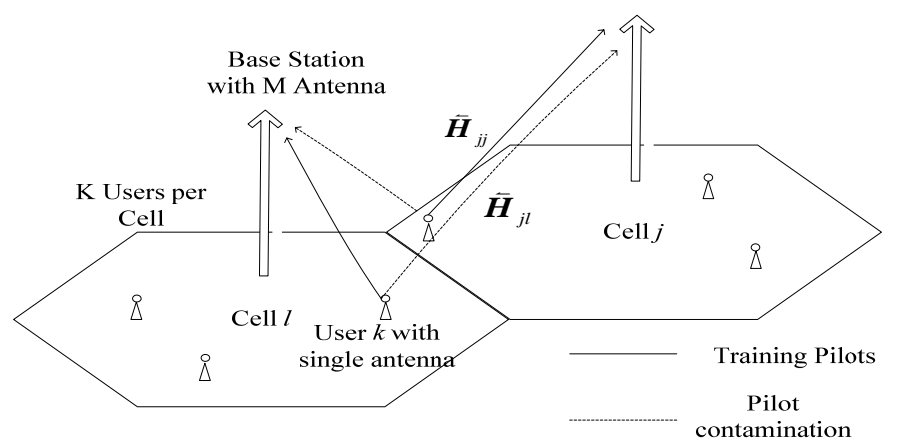

Fig. 1. Uplink system model of Large-scale MIMO

where $p_{r}$ is the transmitting power of base station and $\tau$ is the length of pilot sequence; $\overline{\boldsymbol{H}}_{j j}$ of dimension $M \times N$ is the channel matrix from user $k$ of cell $j$ to the base station of cell $j ; \overline{\boldsymbol{H}}_{j j}=\sqrt{\beta_{j j}} \overline{\boldsymbol{h}}_{j j}$ and $\beta_{j j}$ are indicated as real coefficients of the large scale fading and shadow fading which are assumed to be constant and known to all base stations; $\overline{\boldsymbol{h}}_{j j}$ of dimension $M \times N$ is the fast fading channel matrix whose elements are independent and identically distributed (i.i.d.) with $C N(0,1) ; \overline{\boldsymbol{x}}_{j}$ denotes the training pilots transmitted by the $k$-th user of $L$ cells; $\overline{\boldsymbol{x}}_{i}$ denotes pilots used by cell $i$ and orthogonal with $\overline{\boldsymbol{x}}_{j}$ which can be indicated as $\overline{\boldsymbol{x}}_{i} \overline{\boldsymbol{x}}_{j}^{H}=\delta_{i j}, \delta_{i j}$ denotes the zero matrix; $\overline{\boldsymbol{n}}_{\boldsymbol{j}}$ is the additive white Gaussian noise of cell $j$ whose elements are independent and $\overline{\boldsymbol{n}}_{j} \in C N(0,1)$.

From (1) the LS (Least Square) estimate $\hat{\boldsymbol{H}}_{j j}$ of the channel $\overline{\boldsymbol{H}}_{j j}$, given $\overline{\boldsymbol{y}}_{j}$ is

$$
\hat{\boldsymbol{H}}_{j j}=\overline{\boldsymbol{y}}_{j} \cdot \overline{\boldsymbol{x}}_{j}^{H} / \sqrt{p_{r} \tau}=\overline{\boldsymbol{H}}_{j j}+\sum_{l=1, l \neq j}^{L} \overline{\boldsymbol{H}}_{j l}+\overline{\boldsymbol{n}}_{j}^{\prime},
$$

where $\overline{\boldsymbol{n}}_{j}^{\prime}=\frac{\overline{\boldsymbol{n}}_{j} \overline{\boldsymbol{x}}_{j}^{H}}{\sqrt{p_{r} \tau}}, \overline{\boldsymbol{n}}_{j}^{\prime} \in C N\left(0, \frac{\boldsymbol{I}_{M}}{p_{r} \tau}\right)$. It is shown in equation (2) that the estimated channel $\hat{\boldsymbol{H}}_{j j}$ contains the desired channel $\overline{\boldsymbol{H}}_{j j}$, the pilot pollution channel $\sum_{l=1, l \neq j}^{L} \overline{\boldsymbol{H}}_{j l}$ and the additive white noise $\overline{\boldsymbol{n}}_{j}^{\prime}$. If the downlink precoding directly applies the estimated channel $\hat{\boldsymbol{H}}_{j j}$ to calculate precoding vectors, it would greatly degrade the performance gains of Large-scale MIMO by inaccurate precoding. 


\section{A large-scale MIMO precoding algorithm by interference alignment}

Interference Alignment (IA) mitigates the impact of interference by aligning multiple interference signals in a signal subspace with a dimension smaller than the number of interferers. The transmitting precoding and the receiving precoding is solved in this algorithm. $v_{j}$ is the transmitting precoding matrix of user $k$ of cell $j$.

For the downlink OFDM symbol $t+\Delta t$ and subcarrier $\omega$, the received signal $\boldsymbol{y}_{j}$ of the user $k$ of cell $j$ can be written as

$$
\boldsymbol{y}_{j}=\boldsymbol{H}_{j j} \boldsymbol{v}_{j} x_{j}+\sum_{i=1, i \neq j}^{I} \boldsymbol{H}_{j i} \boldsymbol{v}_{i} \boldsymbol{x}_{i}+\boldsymbol{n}_{j},
$$

where: the first item $\boldsymbol{H}_{j j} \boldsymbol{v}_{j} \boldsymbol{x}_{j}$ is the desired signal from the base station of cell $j$ to the user $k$ of cell $j$, the second item $\sum_{l=1, l \neq j}^{L} \boldsymbol{H}_{j l} \boldsymbol{v}_{l} \boldsymbol{x}_{l}$ is the interference signal from adjacent cells to the user $k$ of cell $l ; \boldsymbol{H}_{j l}$ is the $N \times M$ channel matrix from the base station of cell $l$ to user $k$ of cell $l$, each element of the matrix is independent and identically distributed with $C N(0,1) ; \boldsymbol{n}_{j}$ is the additive white Gaussian noise vector with zero mean unit variance per entry.

The user $k$ of cell $j$ decodes the desired signals coming from their corresponding base station by multiplying the receiving precoding matrix $\boldsymbol{u}_{j}$ and the signal at user $k$ of cell $j$ after the receiver combining is given by

$$
\tilde{\boldsymbol{y}}_{j}=\boldsymbol{u}_{j}^{H} \boldsymbol{y}_{j}=\boldsymbol{u}_{j}^{H} \boldsymbol{H}_{j j} \boldsymbol{v}_{j} \boldsymbol{x}_{j}+\boldsymbol{u}_{j}^{H} \sum_{i=1, i \neq j}^{L} \boldsymbol{H}_{j i} \boldsymbol{v}_{i} \boldsymbol{x}_{i}+\boldsymbol{u}_{j}^{H} \boldsymbol{n}_{j} .
$$

According to the interference alignment theory, in (4), $\operatorname{rank}\left(u_{j}^{H} j v_{j}\right)$ is the rank of the matrix of desired signals and considered as the spatial freedom degree of desired signals, $\operatorname{rank}\left(u_{j}^{H} \sum_{i=1, i \neq j}^{L} H_{j l} v_{i}\right)$ is the rank of the matrix of the interference signal from adjacent cells and considered as an interference signal space. There $\operatorname{rank}(\cdot)$ denotes the rank of the matrix. For the receiving signal in (4), the ideal interference alignment requires that the interference signals and desired signals must satisfy the following conditions:

$$
\begin{gathered}
\operatorname{rank}\left(u_{j}^{H} \sum_{i=1, i \neq j}^{I} H_{j l} v_{i}\right)=0 \quad \forall i \neq j, \\
\operatorname{rank}\left(\boldsymbol{u}_{j}^{H} \boldsymbol{H}_{j j} v_{j}\right)=d .
\end{gathered}
$$

Considering that there is always some interference in the actual situation of a wireless network, (5) and (6) are difficult to be met in general. In order to maximize the system capacity and minimize the interference, the actual scene to achieve interference alignment must satisfy the following conditions in the downlink: 


$$
\begin{gathered}
\min \left[\operatorname{rank}\left(\boldsymbol{u}_{j}^{H} \sum_{i=1, i \neq j}^{I} \boldsymbol{H}_{j i} \boldsymbol{v}_{i}\right)\right] \forall i \neq j, \\
\max \left[\operatorname{rank}\left(\boldsymbol{u}_{j}^{H} \boldsymbol{H}_{j j} \boldsymbol{v}_{j}\right)\right] .
\end{gathered}
$$

In the same way, the uplink interference alignment requirements of the interference signal and useful signal space satisfies the following conditions:

$$
\begin{gathered}
\min \operatorname{rank}\left(\overline{\boldsymbol{u}}_{j}^{H} \sum_{l=1, l \neq j}^{L} \overline{\boldsymbol{H}}_{j l} \overline{\boldsymbol{v}}_{l}\right) \quad \forall l \neq j, \\
\operatorname{rank}\left(\overline{\boldsymbol{u}}_{j}^{H} \overline{\boldsymbol{H}}_{j j} \overline{\boldsymbol{v}}_{j}\right)=d .
\end{gathered}
$$

According to the reciprocity of the channel, if the receiver aligns the desired signal to the space which can achieve the maximal space freedom and the least interference at the corresponding transmitter, the transmitting signal acquires similar gains in the same space [12].

In the system model (4), the downlink SINR of user $k$ in cell $j$ is expressed as follows:

$$
\operatorname{SINR}_{j}=\frac{\left|\boldsymbol{u}_{j}^{H} \boldsymbol{H}_{j j} \boldsymbol{v}_{j}\right|^{2}}{1+\sum_{i=1, i \neq j}^{I}\left|\boldsymbol{u}_{j}^{H} \boldsymbol{H}_{j i} \boldsymbol{v}_{i}\right|^{2}}
$$

Formula (11) takes into account only the adjacent cell interference to user $k$, not considering the impact of the pilot contamination on the performance of the system. In a real system, we only acquire the estimated channel matrix $\hat{\boldsymbol{H}}_{j j}$. In the previous analysis, the estimated channel state information contains the desired channel information and the pilot pollution from other reused pilot cells as denoted in (2). To evaluate the impact of pilot contamination, the desired channel information can be rewritten as follows:

$$
\overline{\boldsymbol{H}}_{j j}=\hat{\boldsymbol{H}}_{j j}-\sum_{l=1, l \neq j}^{L} \overline{\boldsymbol{H}}_{j l}-\overline{\boldsymbol{n}}_{j}^{\prime} .
$$

After the downlink channel $\boldsymbol{H}_{j j}$ is replaced by $\overline{\boldsymbol{H}}_{j j}^{H}=\left(\hat{\boldsymbol{H}}_{j j}-\sum_{l=1, l \neq j}^{L} \overline{\boldsymbol{H}}_{j l}-\overline{\boldsymbol{n}}_{j}^{\prime}\right)^{H}$, the power of the desired signals is calculated by the following expression:

$$
\begin{gathered}
\left|\boldsymbol{u}_{j}^{H} \boldsymbol{H}_{j j} \boldsymbol{v}_{j}\right|^{2}=\left|\boldsymbol{u}_{j}^{H}\left(\hat{\boldsymbol{H}}_{j j}-\sum_{l=1, l \neq j}^{L} \overline{\boldsymbol{H}}_{j l}-\overline{\boldsymbol{n}}_{j}^{\prime}\right)^{H} \boldsymbol{v}_{j}\right|^{2}= \\
=\left|\boldsymbol{u}_{j}^{H} \hat{\boldsymbol{H}}_{j j}^{H} \boldsymbol{v}_{j}\right|^{2}+\left|\boldsymbol{u}_{j}^{H} \sum_{l=1, l \neq j}^{L} \overline{\boldsymbol{H}}_{j l}^{H} \boldsymbol{v}_{j}\right|^{2}+\frac{\boldsymbol{I}}{\sqrt{p_{r} \tau}},
\end{gathered}
$$

where $\left|\boldsymbol{u}_{j}^{H} \hat{\boldsymbol{H}}_{j j}^{H} \boldsymbol{v}_{j}\right|^{2}$ is the power of the desired signal, $\left|\boldsymbol{u}_{j}^{H} \sum_{l=1, l \neq j}^{L} \overline{\boldsymbol{H}}_{j l}^{H} \boldsymbol{v}_{j}\right|^{2}$ is the power of the channel pollution in the downlink because the estimated channel state information is polluted by uplink reused pilots. So SINR in (11) is rewritten as 
follows:

$$
\operatorname{SINR}_{j}=\frac{\left|\boldsymbol{u}_{j}^{H} \overline{\boldsymbol{H}}_{j j}^{H} \boldsymbol{v}_{j}\right|^{2}}{1+\sum_{i=1, i \neq j}^{I}\left|\boldsymbol{u}_{j}^{H} \boldsymbol{H}_{j i} \boldsymbol{v}_{i}\right|^{2}+\left|\boldsymbol{u}_{j}^{H} \sum_{l=1, l \neq j}^{L} \hat{\boldsymbol{H}}_{j l}^{H} \boldsymbol{v}_{j}\right|^{2}} .
$$

Since $\boldsymbol{V}_{j} \boldsymbol{V}_{j}^{H}=\boldsymbol{I}_{M}$,

$$
\operatorname{SINR}_{j}=\frac{\left|\boldsymbol{u}_{j}^{H} \overline{\boldsymbol{H}}_{j j}^{H}\right|^{2}}{1+\sum_{i=1, i \neq j}^{I}\left|\boldsymbol{u}_{j}^{H} \boldsymbol{H}_{j i}\right|^{2}+\left|\boldsymbol{u}_{j}^{H} \sum_{l=1, l \neq j}^{L} \overline{\boldsymbol{H}}_{j l}^{H}\right|^{2}} .
$$

According to (15), the receiving precoding matrix $\boldsymbol{u}_{j}$ must achieve the maximal gain of desired signals, but also minimize the power of pilot pollution and the interference power. The total interference at the receiver due to all undesired transmitters and pilot contamination from other non-orthogonal pilot cells, are given by

$$
\boldsymbol{I}_{j}=\operatorname{Tr}\left[\boldsymbol{u}_{j}^{H} \boldsymbol{B}_{j} \boldsymbol{u}_{j}\right],
$$

$\operatorname{Tr}(\square)$ denotes the trace of the matrix, where

$$
\boldsymbol{B}_{j}=\sum_{l=1, i \neq j}^{L}\left|\overline{\boldsymbol{H}}_{j l}^{H}\right|^{2}+\sum_{i=1, i \neq l}^{I}\left|\boldsymbol{H}_{j i}\right|^{2},
$$

$\boldsymbol{B}_{j}$ is the interference and pilot contamination covariance at user $k$ of cell $j$.

The objective function is designing a receiving precoding matrix $\boldsymbol{u}_{j}$ to reduce the pilot contamination and interference. So the unit matrix $\boldsymbol{u}_{j}$ that maximizes $\mathrm{SINR}_{j}$ is given by

$$
\begin{gathered}
\boldsymbol{u}_{j}=\frac{\left(\boldsymbol{B}_{j}\right)^{-1} \overline{\boldsymbol{H}}_{j j}{ }^{H} \boldsymbol{v}_{j}}{\left\|\left(\boldsymbol{B}_{j}\right)^{-1} \overline{\boldsymbol{H}}_{j j}{ }^{H} \boldsymbol{v}_{j}\right\|}= \\
=\frac{\left(\sum_{l=1, i \neq j}^{L}\left|\overline{\boldsymbol{H}}_{j l}^{H}\right|^{2}+\sum_{i=1, i \neq l}^{I}\left|\boldsymbol{H}_{j i}\right|^{2}\right)^{-1} \overline{\boldsymbol{H}}_{j j}{ }^{H} \boldsymbol{v}_{j}}{\left\|\left(\sum_{l=1, i \neq j}^{L}\left|\overline{\boldsymbol{H}}_{j l}^{H}\right|^{2}+\sum_{i=1, i \neq l}^{I}\left|\boldsymbol{H}_{j i}\right|^{2}\right)^{-1} \hat{\boldsymbol{H}}_{j j}{ }^{H} \boldsymbol{v}_{j}\right\|} .
\end{gathered}
$$

Lemma 1. If $x, y \in C^{N \times M}$ are two independent matrices with distribution $\boldsymbol{C N}\left(0, c \boldsymbol{I}_{N \times M}\right)$, then (referring to [9])

$$
\lim _{M \rightarrow \infty} \frac{\boldsymbol{x}^{H} \boldsymbol{y}}{M}=0 \text { and } \lim _{M \rightarrow \infty} \frac{\boldsymbol{x}^{H} \boldsymbol{x}}{M}=c \boldsymbol{I}_{M} .
$$

According to Lemma1, (12) is rewritten as follows: 


$$
\boldsymbol{u}_{j}=\frac{\left(\sum_{l=1, i \neq j}^{L} \beta_{j l} \boldsymbol{I}_{N}+\sum_{i=1, i \neq l}^{I} \beta_{j i} \boldsymbol{I}_{N}\right)^{-1}\left(\hat{\boldsymbol{H}}_{j j}-\sum_{l=1, l \neq j}^{L} \overline{\boldsymbol{H}}_{j l}-\overline{\boldsymbol{n}}_{j}^{\prime}\right)^{H} \boldsymbol{v}_{j}}{\left\|\left(\sum_{l=1, i \neq j}^{L} \beta_{j l} \boldsymbol{I}_{N}+\sum_{i=1, i \neq l}^{I} \beta_{j i} \boldsymbol{I}_{N}\right)^{-1}\left(\hat{\boldsymbol{H}}_{j j}-\sum_{l=1, l \neq j}^{L} \overline{\boldsymbol{H}}_{j l}-\overline{\boldsymbol{n}}_{j}^{\prime}\right)^{H} \boldsymbol{v}_{j}\right\|} .
$$

As to the reciprocity network, the uplink receiving precoding vectors switch to the downlink transmitting precoding vectors: $\boldsymbol{v}_{j}=\overline{\boldsymbol{u}}_{j}$; similarly the downlink receiving precoding vectors switch to the uplink: $\overline{\boldsymbol{v}}_{j}=\boldsymbol{u}_{j}$. This iterative process is accomplished by the switching process of downlink and uplink. Finally, when the iterative results meet the convergence, we meet the requirements of the precoding matrices of interference alignment.

\section{Numerical results}

In this section, the algorithm proposed (referred to as an Iterative IA algorithm) is evaluated by the performance gain with different numbers of antennas (from 20 up to 60 antennas). The simulation parameters are shown in Table 1.

It is assumed that there are $I=3$ cells in a Large-scale MIMO system. We consider the performance evaluation with severe pilot contamination and without pilot contamination. In the situation with severe pilot contamination, Cell 1 and cell 2 use the same pilot sequences, orthogonal with cell 3 . The large scale fading coefficient of cell 1 is $\beta_{1}=0.2$, the large scale fading coefficient of cell 2 is $\beta_{2}=0.2$, and the large scale fading coefficient of cell 3 is $\beta_{3}=0.2$. In the situation without pilot contamination, the three cells respectively use orthogonal pilot sequences, so that there does not exist pilot contamination among the three cells.

It was compared with a matched-filter algorithm (refer to [2]) and multi-cell MMSE precoding (refer to [8]). The matched-filter algorithm used at the base station in the $j$-th cell is given by $\bar{v}_{j j}=\bar{H}_{j j}^{H} /\left\|\bar{H}_{j j}^{H}\right\|$, and the multi-cell MMSE precoding matrix is calculated based on formula (22) in [8].

Table 1. Simulation parameters

\begin{tabular}{|c|c|}
\hline Simulation parameters & Values \\
\hline Multi-cell & Three cells \\
\hline Large-scale fading coefficient & with severe pilot contamination; without pilot contamination \\
\hline \multirow{2}{*}{ Antennas configuration } & Base station: ULA (Uniform Linear Array), spacing $0.5 \lambda$ \\
\hline & UE 4 antenna ULA, Antenna spacing $4 \lambda$ \\
\hline Transmission power & Uplink $p_{r}=10 \mathrm{~dB}$, Downlink $p_{f}=20 \mathrm{~dB}$ \\
\hline Channel model & Complex Gaussian channel \\
\hline Modulation and coding scheme & Adaptive modulation and coding \\
\hline Channel estimation & LS channel estimation \\
\hline Simulation frames & 5000 Frames \\
\hline
\end{tabular}


We simulate the downlink spectrum efficiency of MF, Multi-cell MMSE precoding and Iterative IA algorithm with different numbers of antenn (Fig. 2). Without pilot contamination, the performance of the proposed algorithm is close to Multi-cell MMSE precoding. The performance of MF algorithm is worse than the above algorithms because it cannot handle the downlink interference to the users of the other two cells. In Fig. 2a, the spectrum efficiency of multi-cell MMSE precoding algorithm and Iterative IA algorithm is almost $2 \%$ higher than MF algorithm, when the number of antennas is 40 .

With severe pilot contamination, it is obvious that multi-cell MMSE precoding and Iterative IA algorithm is better than MF algorithm with the pilot contamination reducing. The performance of MF precoding remains almost unchanged with the number of antennas increasing. It is more obvious in Fig. $2 \mathrm{~b}$ because MF precoding is influenced by the severe pilot contamination. The simulation trend is consistent with the references, that the performance of a Large-scale MIMO is limited by the pilot contamination [2-6].

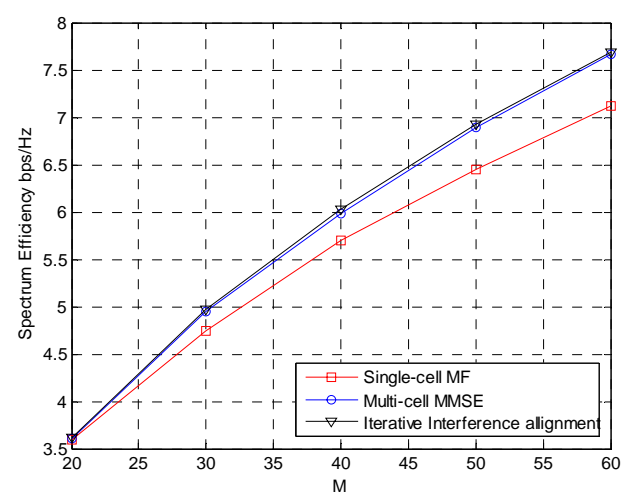

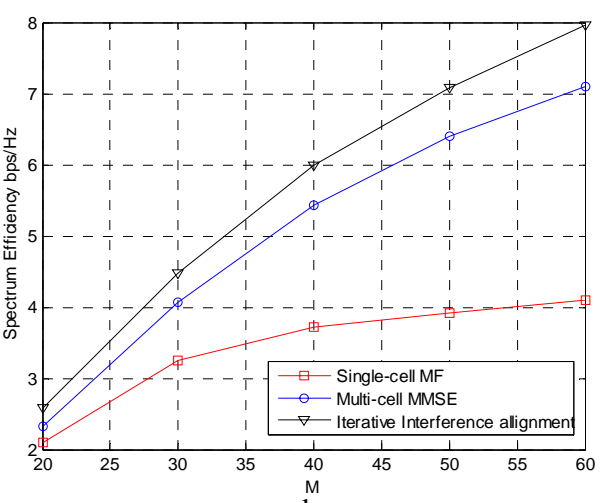

b

Fig. 2. Simulation results of a Large-scale MIMO precoding algorithm: without pilot contamination (a); with severe pilot contamination (b)

In Fig. 2a, the spectrum efficiency of the multi-cell MMSE precoding algorithm is $50 \%$ higher than MF algorithm, and Iterative IA algorithm is $65 \%$ higher than MF algorithm. It was proved that the precoding algorithm reducing pilot contamination could acquire about 1.5 times performance gain than the single-cell MF algorithm in case of severe pilot contamination. Moreover, the Iterative IA can leak the least interference and achieve the biggest space freedom degrees and acquire about $10 \%$ performance gains compared to multi-cell MMSE precoding.

\section{Conclusions}

In this algorithm, Interference alignment is firstly applied for reducing the pilot contamination of a Large-scale MIMO system. The goal of the proposed algorithm is aligning the pilot contamination and inter-cell interference to the same null space in order to acquire the maximal gains in the channel of desired signals. Through several iterations in downlink and uplink transmission, the receiving and 
transmitting precoding vectors are continually optimized based on IA criterion. The simulation results show significant performance gains compared to popular MF algorithm. Moreover, the proposed algorithm improves the performance of the Large-scale MIMO system approximately linearly with the increase of the number of antennas.

Acknowledgment: This paper is sponsored by the National Natural Science Foundation of China (61102047) and the Natural Science Foundation of Shanxi Province (2014JM8320).

\section{References}

1. Marzetta, T. L. Noncooperative Cellular Wireless with Unlimited Numbers of Base Station Antennas. - IEEE Transactions on Wireless Communications, Vol. 9, 2010, No 11, 3590-3600.

2. Rusek, F., D. Persson, Buon Kiong Lau. Scaling Up MIMO: Opportunities and Challenges with Very Large Arrays. - IEEE Signal Processing Magazine, Vol. 30, 2013, No 1, 40-60.

3. Hi e n, Qu o c Ngo, E. G. L a rs s o n, T. L. Marzett. Energy and Spectral Efficiency of Very Large Multiuser MIMO Systems. - IEEE Transactions on Communications, Vol. 61, 2013, No 4, 1436-1449.

4. Jos e, J., A. A s hikh min, T. L. M a r z et t a. Pilot Contamination and Precoding in Multi-Cell TDD Systems. - IEEE Transactions on Wireless Communications, Vol. 10, 2011, No 8, 2640-2651.

5. Hi en, Qu oc Ngo, E. G. Lars o n, T. L. Marzett. Analysis of the Pilot Contamination Effect in Very Large Multicell Multiuser MIMO Systems for Physical Channel Models. - In: Proc. of IEEE International Conference on Acoustics, Speech and Signal Processing, 2011, 3464-3467.

6. Jos e, J., A. A s hikh min, T. L. M arzett a. Pilot Contamination Problem in Multi-Cell TDD Systems. - In: Proc. of IEEE International Symposium on Information Theory, 2009, 21842188.

7. Appaiah, K., A. Ashikhmin, T. L. Marzetta. Pilot Contamination Reduction in MultiUser TDD Systems. - In: Proc. of IEEE International Conference on Communications, 2010, $1-5$.

8. Liangbin, Li, A. A shikhmin, T. L. Marzetta. Pilot Contamination Precoding for Interference Reduction in Large-Scale Antenna Systems. - In: Proc. of 51st Annual Allerton Conference on Communication, Control and Computing, 2013, 226-232.

9. A shikhmin, A., T. L. Marzetta. Pilot Contamination Precoding in Multi-Cell Large-Scale Antenna Systems. - In: Proc. of 2012 IEEE International Symposium on Information Theory, 2012, 1137-1141.

10. Fernandes, F., A. A shikhmin, T. L. Marzetta. Inter-Cell Interference in Noncooperative TDD Large-Scale Antenna Systems. - IEEE Journal on Communications, Vol. 31, 2013, No 2, 192-201.

11. C a d a m b e, V. R., S. A. J a fa r. Interference Alignment and Degrees of Freedom of the K User Interference Channel. - IEEE Transaction on Information Theory, Vol. 54, 2008, No 8, 3425-3441.

12. Go mada m, K., V. R. Cada mbe, S. A. J a far. A Distributed Numerical Approach to Interference Alignment and Applications to Wireless Interference Networks. - IEEE Transactions on Information Theory, Vol. 57, 2008, No 6, 3309-3322.

13. S e o k-H w a n, P a r k, I n k y e L e e. Degrees of Freedom and Sum Rate Maximization for Two Mutually Interfering Broadcast Channels. - In: Proc. of IEEE International Conference on Communications, 2009, 1-5.

14. Changho, Suh, M. Ho, D. Ts e. Downlink Interference Alignment. - Proc. of IEEE Transactions on Communications, Vol. 59, 2011, No 9, 2616-2626. 